\title{
Effect of nutrition education on weight gain and macronutrients intake during pregnancy: a randomized clinical trial
}

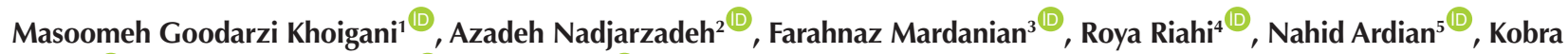

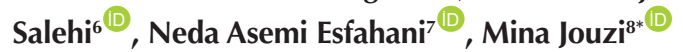

${ }^{1} \mathrm{PhD}$, Child Growth and Development Research Center, Research Institute for Primordial Prevention of Non-communicable Disease, Isfahan University of Medical Sciences, Isfahan, Iran.

${ }^{2}$ Associate Professor, Nutrition and Food Security Research Center, Shahid Sadoughi University of Medical Sciences, Yazd, Iran. Department of Nutrition, School of Public Health, Shahid Sadoughi University of Medical Sciences, Yazd, Iran.

${ }^{3}$ Associate Professor, Department of Obstetrics and Gynecology, School of Medicine, Isfahan University of Medical Sciences, Isfahan, Iran.

${ }^{4} \mathrm{MSc}$ in Biostatistics, Child Department of Pediatrics, Child Growth and Development Research Center, Research Institute for Primordial Prevention of Non-communicable Disease, Isfahan University of Medical Sciences, Isfahan, Iran.

${ }^{5} \mathrm{PhD}$, Social Determinants of Health Research Center, School of Public Health, Shahid Sadoughi University of Medical Sciences, Yazd, Iran.

${ }^{6} \mathrm{PhD}$ Candidate of Reproductive Health, Student Research Committee, Faculty of Nursing and Midwifery, Isfahan University of Medical Sciences, Isfahan, Iran.

${ }^{7}$ School of Nursing and Midwifery, Islamic Azad University, Falavarjan Branch, Isfahan, Iran.

${ }^{8} \mathrm{PhD}$, Nursing and Midwifery Sciences Development Research Center, Najafabad Branch, Islamic Azad University, Najafabad, Iran. Department of Nursing, Najafabad Branch, Islamic Azad University, Najafabad, Iran.

*Corresponding Author: Mina Jouzi, PhD, Nursing and Midwifery Sciences Development Research Center, Najafabad Branch, Islamic Azad University, Najafabad, Iran. Department of Nursing, Najafabad Branch, Islamic Azad University, Najafabad, Iran. Tel: +98-3142292208, 09132334730, Fax: +98-3142291112, Email: minajouzi@pnu.iaun.ac.ir

\begin{abstract}
Background and aims: The imbalance between energy intake and consumption has been recognized as an important factor contributing to the gestational weight gain (GWG). Additionally, the determination of the effect of macronutrient composition on GWG has been recommended by researchers. Therefore, we examined the effect of nutrition education on weight gain, energy, and energy-adjusted macronutrients intake during three trimesters of pregnancy which have not been widely studied.

Methods: This randomized single-blinded clinical trial was performed on 192 primiparous pregnant women in Isfahan, Iran, from May 2015 to September 2016. Data were collected using a questionnaire completed through interviews with pregnant women and prenatal care-related records. We developed an individualized calorie-appropriate diet for each participant at enrollment and then three 45-60 minute training sessions were held at 6-10, 18, and 26 weeks of pregnancy and the subjects' weights were measured during these sessions. A consecutive 3-day food intake record at 6-10, 18 and 34-36 weeks was obtained from two groups to determine the macronutrients. Moreover, physical activity scores were estimated before and after the intervention.

Results: The mean weights at 18,26 , and 40 weeks of gestation were significantly lower in the intervention group $(P=0.024, P=0.040$, and $P=0.015$, respectively). The total energy, energy-adjusted carbohydrate and protein intake at $6-10,18$, and 34-36 weeks of gestation were not significantly different between the two groups $(P>0.05)$. However, the mean energy-adjusted fat intake decreased $(P=0.043)$. In addition, the mean energy-adjusted dietary fiber intake was significantly higher in the intervention group $(P=0.001)$.

Conclusion: In the present individualized calorie-appropriate trial, the mean weights decreased during three trimesters in the intervention group. Additionally, calorie, energy-adjusted carbohydrate and protein intake did not change after intervention while energy-adjusted fat intake decreased.

Keywords: Nutritional intervention, Pregnancy, Macronutrients, Weight gain, Clinical trial, Iran
\end{abstract}

Received: 13 August 2019, Accepted: 4 November 2019, ePublished: 29 April 2020

(C) 2020 The Author(s); Published by Shahrekord University of Medical Sciences. This is an open-access article distributed under the terms of the Creative Commons Attribution License (http://creativecommons.org/licenses/by/4.0), which permits unrestricted use, distribution, and reproduction in any medium, provided the original work is properly cited. 


\section{Introduction}

A balanced and varied diet that contains adequate amounts of energy and nutrients is essential to maintain good maternal health and gestational weight gain (GWG) which affect fetal health and birth weight (1). Excessive GWG is one of the most common complications with an overall prevalence of $18.5-38.3 \%$ (2) and a prevalence of $20.13 \%-37.27 \%$ in Iranian obese women (3). It could result in post-partum weight retention, obesity, gestational diabetes, hypertensive disorders of pregnancy, cardiometabolic outcomes in women, and obesity in children (4). Researchers have recognized the imbalance between energy intake and consumption as an important factor contributing to the GWG (5). In this regard, the determination of the effect of macronutrient composition on this problem has been recommended (6). The nutrition intervention used by Wolff et al restricted the energy intake and the GWG (7). Decreased daily intake of calorie, fat, saturated fat, and cholesterol were detected after another intervention which reduced EGWG (8). In our trial, we prevented GWG by nutrition education (9) which was the first nutritional intervention to the best of our knowledge since other successful trials included nutrition and physical activity trials. In this study, we evaluated the effect of nutrition education on weight gain during three trimesters of pregnancy. Another aim of the current study was to assess the effect of education on the energy and energyadjusted macronutrient intake.

\section{Materials and Methods}

This randomized clinical trial (identifier: IRCT2016012026129N1; https://en.irct.ir/trial/21740) was performed among 192 primiparous pregnant women in Isfahan, Iran, from May 2015 to September 2016. The sample size was calculated by the following formula:

$\mathrm{n}=[(\mathrm{Z} 1-\alpha / 2+\mathrm{Z} 1-\beta) 2 \cdot 2 \mathrm{~S} 2] / \Delta 2$, based on a significance level of $5 \%$, a power of at least $80 \%$, and a standard deviation of 7 for at least a $3 \mathrm{~kg}$ difference in the gained weight between two groups. An attrition rate of $10 \%$ was considered and 96 participants were determined for each group. In this study, 5 hospitals, 15 community health centers, and 15 private offices were selected according to the stratified sampling. First, Iranian women with 18 years of age and older, a body mass index (BMI) of lower than 40 $\mathrm{kg} / \mathrm{m}^{2}$, and a gestational age of 6-10 weeks were included in the study. Exclusion criteria were weight-related complications (10), anemia, urinary tract complications, use of a special regimen (11), a history of diabetes (12), mental diseases (13), chronic disease, and addiction (14).

Gynecologists medically prescreened females and then a person in charge explained the study objectives to them. After providing the written consent to participate in the study, the participants were randomly classified into groups. Computer-generated codes were determined by random allocation software (version 1.0.0) and then sealed in consecutively numbered opaque envelopes. The participants were then randomized by opening the next sealed envelope to assign them in the intervention or control group to complete the sample size. Routine prenatal care was provided for all participants. In addition, training sessions were held for the intervention group. Gynecologists and midwives were blind to the participants randomization and the educational content. However, we could not blind the instructor and pregnant women due to the nature of the study.

\section{Instrument}

Participants' characteristics were collected by a questionnaire completed through interviews with pregnant women and prenatal care-related records. Since these records were prepared in standardized forms, their reliability had already been confirmed. In addition, patient records were completed by experts, which ensured their reliability.

A three-day dietary recall including one weekend day (15) was used to measure the food intake at 6-10 and 35 weeks of gestation. The obtained data were analyzed for nutrients using the nutritionist IV software while the person in charge was blind to study objectives. The Pregnancy Physical Activity Questionnaire was used to measure the participants' physical activity before and after the intervention (16).

\section{Nutrition education intervention}

Our intervention was planned for the intervention group considering the initial evaluation (15). A nutritionist developed an individualized calorie-appropriate diet for each participant at enrollment. Three 45-60 minute training sessions were then held at 6-10, 18, and 26 weeks of pregnancy. In the first session, an educational booklet (17) including the benefits of trained items, the barriers to using them, and the solutions for overcoming the barriers was given to each participant in the intervention group. Educational content included the following main points: (1) varied and balanced diet according to the five food groups of the food pyramid including grain, milk, meat, vegetables, and fruit expressed as servings/day; (2) GWG according to the IOM guidelines; and (3) healthy eating. Pregnant mothers were advised to eat low-fat dairy products, high fiber bread instead of white bread, and vegetables instead of pickles. Energy intake was estimated according to the initial dietary evaluation and 50\% to 55\% of that was allocated to the total carbohydrate intake. The participants were advised to take complex carbohydrates instead of simple carbohydrates. Approximately 25\% to $30 \%$ of energy intake was allocated to the total fat intake and $15 \%$ to $20 \%$ to protein intake. We made any attempts to increase the monounsaturated fatty acids and decrease the saturated and trans fatty acids. Fluid and micronutrient needs were determined according to the national guideline. The intervention group recorded their daily food intake for 
one month to increase their self-efficacy and to test their compliance. In addition, the instructions were explained to the mothers in the intervention group in the second session in order to increase their self-efficacy (goal-setting techniques). We created groups of two to eight persons during the second and third sessions to perform group discussion, role-playing, and brainstorming strategies. Two telephone numbers were also given to participants so that they could ask their questions and their potential stress could be managed. Computer-based programs were used to educate healthy cooking methods during the third session. The first author instructed the pregnant mothers. The participants' weights were estimated using a digital scale at each session during 34-36 and 40 weeks of pregnancy.

\section{Statistical analysis}

Statistical analyses were performed using SPSS version 18.0 (IBM Company, USA). Independent $t$ test and $\chi^{2}$ were used to determine the homogeneity of the baseline characteristics in two groups. The Kolmogorov-Smirnov test was used to analyze the normal distribution of the data, and significance level was considered $5 \%$. We compared the mean weights at $6-10,18,26,34-36$, and 40 weeks of gestation using repeated measures analysis of variance. Macronutrients values and dietary fiber intake were adjusted for total energy intake by the residuals method as described by Willett and Stampfer (18). The mean values of total energy and energy-adjusted macro-nutrients intake were compared by repeated measurement ANOVA at 18, and 34-36 weeks of gestation and over time (during pregnancy).

\section{Results}

The participants' demographic characteristics were not significantly different between two groups (Table 1). The participants' mean weights were significantly lower at 18 , 26 , and 40 weeks of gestation in the intervention group (Table 2). The mean value of total GWG was significantly lower in intervention group $(P=0.01)$. Table 3 shows the comparison of mean values of energy and energy-adjusted macro-nutrient intake between two groups at 6-10, 26, and 34-36 weeks of gestation. Moreover, the comparison of these variables was done over time (the last column of Table 3). The results of repeated measures ANOVA showed that the fiber intake enhanced after intervention $(\mathrm{P}=0.001)$.

\section{Discussion}

In the present trial, the mean weight gain decreased in the intervention group during three trimesters. After intervention, total energy, energy-adjusted protein, and carbohydrate intake were not different between two groups. However, energy-adjusted dietary fiber intake increased and energy-adjusted fat intake decreased significantly.

The mean weights at 18,26 , and 40 weeks of gestation decreased in intervention group. There are few studies that
Table 1. Comparison of Participants' Characteristics and Baseline Values in Study Groups $(\mathrm{n}=192)$

\begin{tabular}{|c|c|c|c|}
\hline Variable & $\begin{array}{c}\text { Intervention } \\
\quad(\mathrm{N}=\mathbf{8 6})\end{array}$ & $\begin{array}{l}\text { Control } \\
(\mathrm{N}=88)\end{array}$ & $P$-value \\
\hline Age (years) & $26.58 \pm 3.80$ & $26.71 \pm 4.04$ & $0.83^{*}$ \\
\hline Pregravid weight (kg) & $63.29 \pm 11.85$ & $61.14 \pm 10.04$ & $0.17^{*}$ \\
\hline Pregravid BMI $\left(\mathrm{kg} / \mathrm{m}^{2}\right)$ & $23.70 \pm 4.07$ & $22.32 \pm 3.91$ & $0.99 *$ \\
\hline Years of education & 14.62 & 14.15 & 0.66 \\
\hline \multicolumn{4}{|l|}{ Family income (Rials) } \\
\hline$<6000000$ & $24(27.06 \%)$ & $138(11.04 \%)$ & \multirow{3}{*}{$0.66^{* *}$} \\
\hline $6000000-12000000$ & $44.94(59 \%)$ & $59(62.11 \%)$ & \\
\hline$>12000000$ & $25(28.09 \%)$ & $16(16.85 \%)$ & \\
\hline $\begin{array}{l}\text { Physical activity (met/hour) in } \\
\text { the first trimester }\end{array}$ & $31.02 \pm 11.55$ & $30.01 \pm 11.02$ & $0.61^{*}$ \\
\hline $\begin{array}{l}\text { Physical activity (met/hour) in } \\
\text { the third trimester }\end{array}$ & $29.92 \pm 10.78$ & $27.89 \pm 10.50$ & $0.54^{*}$ \\
\hline
\end{tabular}

have reported the weight of pregnant mothers at different stages of pregnancy in all BMI groups. For example, the intervention used by Wolff et al restricted the GWG in obese women who received 10 consultations of 1 hour, each with a trained dietitian, during the pregnancy (7). Moreover, the intervention used by Sun et al at weeks 8-12 and every month in the second trimester prevented excessive GWG in overweight and obese women (19). In another study, obese women received group-based dietary intervention after their second individualized session and throughout pregnancy which resulted in lower GWG (20). In a report by Robertson and Ladlow, three or more interventional sessions were significantly more efficient compared with the initial assessment only (21). However, Bechtel-Blackwell conducted computer-assisted selfinterview and reported that nutrition education decreased GWG in the first trimester while maternal weights were significantly greater during the second and third trimesters (22). In another dietary intervention, pregnant women were provided with recommendations on the basis of the Australian guideline by a dietitian (23). Besides that, individualized advice regarding weight gain and nutrient targets was provided. Diet quality improved in the study, while GWG did not change. It seems that training sessions during pregnancy along with the strategies used in this study and similar surveys were able to control GWG.

In our trial, the mean energy intake at 6-10, 18, and 34-36 weeks of gestation were not significantly different between two groups. However, the intervention used by Hui et al (8) decreased energy intake in participants of all BMI groups and resulted in a reduction in GWG. Additionally, the intervention used by Wolf et al restricted calorie and decreased GWG in obese women (7). Consistently, a systematic review of observational surveys suggested that GWG might be decreased by lower energy intake in pregnancy (24). Here we note that the intervention (8) used by Hui et al lasted eight weeks while the intervention in the present study lasted 30-34 weeks and reduced energy 
Table 2. The Comparison of the Participants' Mean Weights at 6-10, 18, 26, 34-36, and 40 weeks of Gestation by Repeated Measurement ANOVA

\begin{tabular}{|c|c|c|c|c|c|c|}
\hline \multirow{2}{*}{ Time } & \multirow{2}{*}{ Group } & \multirow{2}{*}{ Mean } & \multirow{2}{*}{ Std. Error } & \multicolumn{2}{|c|}{$95 \%$ Confidence interval } & \multirow{2}{*}{ P-value } \\
\hline & & & & Lower bound & Upper bound & \\
\hline \multirow{2}{*}{ 6-10 week } & 1 & 62.45 & 0.26 & 61.93 & 62.97 & \multirow{2}{*}{0.052} \\
\hline & $\mathrm{C}$ & 63.19 & 0.27 & 62.66 & 63.72 & \\
\hline \multirow{2}{*}{18} & 1 & 64.70 & 0.39 & 63.92 & 65.47 & \multirow{2}{*}{0.024} \\
\hline & $\mathrm{C}$ & 65.98 & 0.40 & 65.19 & 66.77 & \\
\hline \multirow{2}{*}{26} & 1 & 69.19 & 0.43 & 68.34 & 70.03 & \multirow{2}{*}{0.040} \\
\hline & $\mathrm{C}$ & 70.46 & 0.44 & 69.59 & 71.32 & \\
\hline \multirow{2}{*}{$34-36$} & 1 & 73.22 & 0.46 & 72.31 & 74.14 & \multirow{2}{*}{0.050} \\
\hline & C & 74.53 & 0.47 & 73.60 & 75.46 & \\
\hline \multirow{2}{*}{40} & 1 & 74.93 & 0.49 & 73.95 & 75.90 & \multirow{2}{*}{0.015} \\
\hline & C & 76.65 & 0.50 & 75.66 & 77.64 & \\
\hline \multicolumn{4}{|c|}{ Time*Group } & \multicolumn{2}{|c|}{ F statistics : 1.44} & 0.22 \\
\hline
\end{tabular}

I: intervention

C: control

Table 3. The Mean Values of Energy and Energy-adjusted Macronutrients at 18 and 34-36 Weeks of Pregnancy in Two Groups

\begin{tabular}{|c|c|c|c|c|c|c|c|c|}
\hline \multirow{2}{*}{ Variable } & \multirow{2}{*}{\multicolumn{2}{|c|}{ Time group }} & \multirow{2}{*}{ Mean } & \multirow{2}{*}{ Std. Error } & \multicolumn{2}{|c|}{$95 \%$ Confidence interval } & \multirow{2}{*}{ P-value* } & \multirow{2}{*}{ P-value** } \\
\hline & & & & & Lower bound & Upper bound & & \\
\hline \multirow{4}{*}{ Energy } & 18 & 1 & 1840.57 & 33.46 & 1774.18 & 1906.96 & \multirow{2}{*}{0.72} & \multirow{4}{*}{0.723} \\
\hline & C & & 1859.48 & 40.53 & 1779.08 & 1939.89 & & \\
\hline & $34-36$ & 1 & 1892.74 & 66.93 & 1759.97 & 2025.52 & \multirow{2}{*}{0.72} & \\
\hline & $\mathrm{C}$ & & 1930.58 & 81.05 & 1769.77 & 2091.39 & & \\
\hline \multirow{4}{*}{ Protein } & 18 & 1 & 69.023 & 1.493 & 66.063 & 71.983 & \multirow{2}{*}{0.47} & \multirow{4}{*}{0.464} \\
\hline & $\mathrm{C}$ & & 67.273 & 1.857 & 63.591 & 70.956 & & \\
\hline & $34-36$ & 1 & 74.949 & 2.985 & 69.029 & 80.869 & \multirow{2}{*}{0.46} & \\
\hline & $\mathrm{C}$ & & 71.449 & 3.714 & 64.084 & 78.814 & & \\
\hline \multirow{4}{*}{ Fat } & 18 & I & 52.687 & 1.203 & 50.301 & 55.074 & \multirow{2}{*}{0.04} & \multirow{4}{*}{0.043} \\
\hline & C & & 56.755 & 1.526 & 53.728 & 59.782 & & \\
\hline & $34-36$ & 1 & 50.631 & 2.406 & 45.857 & 55.404 & \multirow{2}{*}{0.04} & \\
\hline & C & & 58.767 & 3.051 & 52.713 & 64.820 & & \\
\hline \multirow{4}{*}{ Carbohydrate } & 18 & 1 & 275.497 & 5.922 & 263.750 & 287.244 & \multirow{2}{*}{0.96} & \multirow{4}{*}{0.963} \\
\hline & C & & 275.056 & 7.273 & 260.630 & 289.481 & & \\
\hline & $34-36$ & I & 287.895 & 11.845 & 264.401 & 311.389 & \multirow{2}{*}{0.90} & \\
\hline & C & & 287.012 & 14.546 & 258.160 & 315.864 & & \\
\hline
\end{tabular}

I: intervention

C: control

$P$-value*: The comparison of mean values of energy and energy-adjusted macronutrient intake in two groups at 6-10, 26 and 34-36 weeks of gestation using repeated measures ANOVA

$P$-value**: The comparison of mean values of energy and energy-adjusted macronutrient intake by repeated measures ANOVA over time

intake, which could be associated with low birth weight. A reduction in energy intake in studies that were conducted on overweight and obese women can make sense while our participants were selected from all BMI groups. In addition, we individualized calorie intake and the reduction in energy intake was not our aim. Thomas et al reported that their energy-balance model could make predictions about the relationship between energy consumption and weight gain during pregnancy (25).

After the intervention, energy-adjusted carbohydrate intake was not different between the groups in the present study. The findings reported by Guelinckx et al were similar to our results (26); however, in a study by Hui et al, carbohydrate intake decreased in normal women, but not in overweight and obese subjects (8). In a study by Mottola et al, the intervention reduced carbohydrate intake in overweight and obese participants (27). Although energyadjusted carbohydrate intake was not significantly different between the two groups in the present study, changes in carbohydrate intake might be the reason for weight control in the intervention group as energy-adjusted dietary fiber intake enhanced significantly after intervention.

Comparison of energy-adjusted protein intake did not show a significant difference between two groups which is contrary to other studies in overweight and obese women (27) or in all BMI groups $(8,28)$. The protein intake increased after the intervention in the present study and the lack of the significant difference between the two groups was due to the education of the control group by their health care providers as we observed that both groups had enhanced energy-adjusted protein intake.

Energy-adjusted fat intake decreased during pregnancy in experimental group which is consistent with the study conducted by Hui et al, in which the intervention decreased GWG in all BMI groups (8). Reduced fat intake in experimental group after these interventions is a logical finding as GWG did not decrease in one study in which lower fat intake was not observed after intervention (26).

The strengths of this study are the early enrollment of participants, thelongitudinal design, repeated measurements 
of the same variables during pregnancy, and measurements of macronutrient intake in addition to weight gain. Our study showed that continuing nutrition education during pregnancy is effective in improving nutrient intake and weight control. However, the intervention in the present study was performed on a rather small sample of thin and obese women. Therefore, the replication of the study on thin, normal, overweight, and obese women with an adequate sample size is recommended.

\section{Conclusion}

In the present individualized calorie-appropriate trial during three trimesters, the mean weight decreased in intervention group. Moreover, calorie, energy-adjusted carbohydrate, and protein intake did not change after intervention while energy-adjusted fat intake decreased.

Conflict of Interests

Authors declare no conflicts of interests.

\section{Ethical Approval}

This study protocol was approved by Research Ethics Committee for Health Sciences of Public Health School of Shahid Sadoughi University of Medical Sciences (4326) and the Vice Chancellery of Research and Technology of Isfahan University of Medical Sciences (294048).

\section{Authors' Contributions}

$\mathrm{M} \mathrm{G-KH}$ and AN conceived and designed the trial. AN, FM, N A, $\mathrm{K} \mathrm{S}$ and $\mathrm{MJ}$ reviewed and edited the manuscript. FM introduced the pregnant women. $\mathrm{M} \mathrm{G-KH}$ and $\mathrm{KS}$ performed the educational sessions. RR analyzed the data. M G-KH and M J wrote the paper. $N$ $\mathrm{A}$ and $\mathrm{N}$ A-E gathered the data.

\section{Funding/Support}

This trial was financially supported by Public Health College of Shahid Sadoughi University of Medical Sciences (4326).

\section{References}

1. Siega-Riz AM, King JC. Position of the American Dietetic Association and American Society for Nutrition: obesity, reproduction, and pregnancy outcomes. J Am Diet Assoc. 2009;109(5):918-27. doi: 10.1016/j.jada.2009.03.020.

2. Goldstein RF, Abell SK, Ranasinha S, Misso ML, Boyle JA, Harrison $\mathrm{CL}$, et al. Gestational weight gain across continents and ethnicity: systematic review and meta-analysis of maternal and infant outcomes in more than one million women. BMC Med. 2018;16(1):153. doi: 10.1186/s12916-018-1128-1.

3. Delvarian Zadeh M, Ebrahimi H, Bolbol Haghighi N. Surveying pregnant women's nutritional status and some factors affecting it in cases referring to Shahrood health-care centers. Journal of Birjand University of Medical Sciences. 2006;13(4):42-9. [Persian].

4. Phelan S, Phipps MG, Abrams B, Darroch F, Schaffner A, Wing RR. Randomized trial of a behavioral intervention to prevent excessive gestational weight gain: the Fit for Delivery Study. Am J Clin Nutr. 2011;93(4):772-9. doi: 10.3945/ajcn.110.005306.

5. Gardner B, Wardle J, Poston L, Croker H. Changing diet and physical activity to reduce gestational weight gain: a metaanalysis. Obes Rev. 2011;12(7):e602-20. doi: 10.1111/j.1467789X.2011.00884.x.

6. Tielemans MJ, Garcia AH, Peralta Santos A, Bramer WM,
Luksa N, Luvizotto MJ, et al. Macronutrient composition and gestational weight gain: a systematic review. Am J Clin Nutr. 2016;103(1):83-99. doi: 10.3945/ajen.115.110742.

7. Wolff S, Legarth J, Vangsgaard K, Toubro S, Astrup A. A randomized trial of the effects of dietary counseling on gestational weight gain and glucose metabolism in obese pregnant women. Int J Obes (Lond). 2008;32(3):495-501. doi: 10.1038/sj.ijo.0803710.

8. 8 Hui A, Back L, Ludwig S, Gardiner P, Sevenhuysen G, Dean $\mathrm{H}$, et al. Lifestyle intervention on diet and exercise reduced excessive gestational weight gain in pregnant women under a randomised controlled trial. Bjog. 2012;119(1):70-7. doi: 10.1111/j.1471-0528.2011.03184.x.

9. Mazloomy Mahmoodabad SS, Baghiani Moghadam $\mathrm{MH}$, Nadjarzadeh A, Mardanian F, Mohammadi R, Zare N, Rejali Z, Goodarzi-khoigani M. The Effect of Nutrition Education on Gestational Weight Gain based on the Pender's Health Promotion Model: A Randomized Clinical Trial Study. Journal Of Isfahan Medical School. 2020;37(552):1272-1279.

10. Muktabhant B, Lawrie TA, Lumbiganon P, Laopaiboon M. Diet or exercise, or both, for preventing excessive weight gain in pregnancy. Cochrane Database Syst Rev. 2015(6):CD007145. doi: 10.1002/14651858.CD007145.pub3.

11. Brantley SL, Russell MK, Mogensen KM, Wooley JA, Bobo E, Chen Y, et al. American Society for Parenteral and Enteral Nutrition and Academy of Nutrition and Dietetics: revised 2014 standards of practice and standards of professional performance for registered dietitian nutritionists (competent, proficient, and expert) in nutrition support. J Acad Nutr Diet. 2014;114(12):2001-8.e37. doi: 10.1016/j.jand.2014.08.018.

12. Herring SJ, Oken E, Rifas-Shiman SL, Rich-Edwards JW, Stuebe AM, Kleinman KP, et al. Weight gain in pregnancy and risk of maternal hyperglycemia. Am J Obstet Gynecol. 2009;201(1):61.e1-7. doi: 10.1016/j.ajog.2009.01.039.

13. Lee HS. The factors influencing health-related quality of life in the elderly: focused on the general characteristics, health habits, mental health, chronic diseases, and nutrient intake status: data from the Fifth Korea National Health and Nutrition Examination Survey (KNHANES V), 2010 2012. Korean J Community Nutr. 2014;19(5):479-89. doi: 10.5720/ kjcn.2014.19.5.479.

14. Althuizen E, van Poppel MN, Seidell JC, van der Wijden C, van Mechelen W. Design of the new life(style) study: a randomised controlled trial to optimise maternal weight development during pregnancy. [ISRCTN85313483]. BMC Public Health. 2006;6:168. doi: 10.1186/1471-2458-6-168.

15. Yang YJ, Kim MK, Hwang SH, Ahn Y, Shim JE, Kim DH. Relative validities of 3-day food records and the food frequency questionnaire. Nutr Res Pract. 2010;4(2):142-8. doi: 10.4162/ nrp.2010.4.2.142.

16. Chasan-Taber L, Schmidt MD, Roberts DE, Hosmer D, Markenson G, Freedson PS. Development and validation of a pregnancy physical activity questionnaire. Med Sci Sports Exerc. 2004;36(10):1750-60. doi: 10.1249/01. mss.0000142303.49306.0d.

17. Bakhshandeh M, Pooraram H, Torkestani F, Torabi P, Abedini MD. The national comprehensive guildline for mothers: an eating guide with practical educational points specifically developed to promote healthy eating during pregnancy and breast feeding. Tehran; 2013.

18. Willett W, Stampfer MJ. Total energy intake: implications for epidemiologic analyses. Am J Epidemiol. 1986;124(1):17-27. doi: 10.1093/oxfordjournals.aje.a114366.

19. Sun $\mathrm{Y}$, Zhao $\mathrm{H}$. The effectiveness of lifestyle intervention in early pregnancy to prevent gestational diabetes mellitus in 
Chinese overweight and obese women: a quasi-experimental study. Appl Nurs Res. 2016;30:125-30. doi: 10.1016/j. apnr.2015.10.006.

20. Vesco KK, Karanja N, King JC, Gillman MW, Leo MC, Perrin $\mathrm{N}$, et al. Efficacy of a group-based dietary intervention for limiting gestational weight gain among obese women: a randomized trial. Obesity (Silver Spring). 2014;22(9):1989-96. doi: 10.1002/oby.20831.

21. Robertson N, Ladlow B. Effect of individual dietetic intervention on gestational weight gain and associated complications in obese pregnant women. Aust N Z J Obstet Gynaecol. 2018;58(3):274-7. doi: 10.1111/ajo.12711.

22. Bechtel-Blackwell DA. Computer-assisted self-interview and nutrition education in pregnant teens. Clin Nurs Res. 2002;11(4):450-62. doi: 10.1177/105477302237456.

23. Opie RS, Neff M, Tierney AC. A behavioural nutrition intervention for obese pregnant women: Effects on diet quality, weight gain and the incidence of gestational diabetes. Aust N Z J Obstet Gynaecol. 2016;56(4):364-73. doi: 10.1111/ ajo.12474.

24. Streuling I, Beyerlein A, Rosenfeld E, Schukat B, von Kries R. Weight gain and dietary intake during pregnancy in industrialized countries--a systematic review of observational studies. J Perinat Med. 2011;39(2):123-9. doi: 10.1515/ jpm.2010.127.

25. Thomas DM, Navarro-Barrientos JE, Rivera DE, Heymsfield SB, Bredlau C, Redman LM, et al. Dynamic energy-balance model predicting gestational weight gain. Am J Clin Nutr. 2012;95(1):115-22. doi: 10.3945/ajcn.111.024307.

26. Guelinckx I, Devlieger R, Mullie P, Vansant G. Effect of lifestyle intervention on dietary habits, physical activity, and gestational weight gain in obese pregnant women: a randomized controlled trial. Am J Clin Nutr. 2010;91(2):373-80. doi: 10.3945/ajcn.2009.28166.

27. Mottola MF, Giroux I, Gratton R, Hammond JA, Hanley A, Harris $S$, et al. Nutrition and exercise prevent excess weight gain in overweight pregnant women. Med Sci Sports Exerc. 2010;42(2):265-72. doi: 10.1249/MSS.0b013e3181b5419a.

28. Aşcı Ö, Rathfisch G. Effect of lifestyle interventions of pregnant women on their dietary habits, lifestyle behaviors, and weight gain: a randomized controlled trial. J Health Popul Nutr. 2016;35:7. doi: 10.1186/s41043-016-0044-2. 